\title{
Windfelduntersuchung in Zürich: Ergebnisse von Messungen und numerischen Modellierungen
}

\begin{abstract}
Summary
From 1985 to 1989 the Department of Geography of the ETH in Zurich carried out a project called "Stadtklima Zürich". Following a brief description of the project the paper is centered on theoretical and experimental studies concerning the airflow above Zurich.

The wind measurements were undertaken with a network of about 20 stations. The interpretation of these measurements was concentrated on important weather types. Apart from a cartographic representation of the measured data, a diagnostic model (DIAMO) was used for the data interpolation. A non-hydrostatic numerical model (MONARC) helped to interprete dynamical processes over complex topography. With an example of a situation with easterly synoptic wind the measured windfield is compared with the simulations which show a fairly good correspondence. Measurements over the railroad tracks near the Central Station show the transition from mesoscale topographic to microscale or urban building effects. By using a particle trajectory model (LADI and TRAMO), the problem of air pollution dispersion over the complex urban topography is discussed.
\end{abstract}

\section{Einleitung}

Das Geographische Institut der ETH führte in den Jahren 1985 bis 1989 in Zürich ein stadtklimatologisches Projekt durch. Nebst der logistischen Nähe wurde die Stadt Zürich als Untersuchungsgebiet gewählt, weil es sich um die größte Schweizer Stadt handelt, in der sich insbesondere tagsüber sehr viele Menschen auf engem Raum aufhalten. Die Schadstoffbelastung der Luft ist seit Jahren sehr ausgeprägt.

In den Voruntersuchungen zur Projektformulierung wurden mit verschiedenen städtischen und kantonalen Amtsstellen Projektabsicht und Bedürfnisse der Praxis diskutiert. Dabei zeigte sich, daß klimatologische Daten nur spärlich und punktuell vorlagen. Es fehlten damit wichtige Grundlagen für Fragen der Schadstoffausbreitung und der Mikroklimatologie. Dieser Mangel wurde bei Umweltverträglichkeitsuntersuchungen, lufthygienischer Maßnahmenplanung sowie Aspekten der Raumplanung offenkundig. Neben dem Forschungsinteresse lag somit für das Gebiet der Stadt Zürich ein großer Anwendungsbedarf vor.

Finanziert wurde das stadtklimatologische Projekt vom Schweizerischen Nationalfonds zur Förderung der wissenschaftlichen Forschung und von der ETH Zürich. Die Schweizerische Meteorologische Anstalt Payerne (Sek- tion Umweltmeteorologie) leistete in Form einer technischen Zusammenarbeit ebenfalls einen wesentlichen Beitrag.

\section{Ziele und Bestandteile des Projektes STADTKLIMA Zürich}

Windfeld und Turbulenz der Atmosphäre - beides Schlüsselgrößen für die Schadstoffausbreitung - sowie Mikroklima sind vom Energieangebot abhängig. Der Titel des Projektes lautete denn auch «Energiebilanz und Mikroklima der untersten atmosphärischen Schicht über der Stadt Zürich». Mit zwei Dissertationen lag der Schwerpunkt des Projektes auf den Untersuchungen zur Turbulenz (ROTACH, 1990) und zum mittleren Windfeld (SCHUHMACHER, 1991). In Form von Diplomarbeiten wurden vor und während des Projektes zu weiteren Themenkreisen Untersuchungen durchgeführt, so zu den Komponenten der Strahlungsbilanz (GYSEL, 1984), zur UV-Strahlung (HOFER, 1985), zur Albedo (ROTH, 1985), zur Strahlungsmodellierung im Straßencanyon (LEUTEN. EGGER, 1988), zur Bestimmung der Grenzschichthöhe (BENZ, 1988) und zum vertikalen Windprofil (SCHÄDLER, 1988).

Im Folgenden wird über die Windfelduntersuchungen berichtet.

\section{Windfeldmessung}

Zur Bestimmung des mittleren Windfeldes wurde ein eigenes Meßnetz installiert, das etwa 20 Stationen umfaßte (Fig.1). Die Messungen erfolgten grundsätzlich über Dachniveau, wozu geeignete Liegenschaften mit Flachdach und einem Liftaufbau gesucht wurden, die etwa die mittlere Höhe der umliegenden Häuser im Quartier aufweisen. Die Meßfühler kamen damit auf etwa 5 Meter über Dachniveau zu liegen.

Die Meßperiode wurde nach der Projektformulierung auf die Wintermonate November bis März der Jahre 1985/86 und 1986/87 gelegt. Mit einem reduzierten

Peter Schuhmacher, Dr. sc. nat. ETH, Geographisches Institut der ETH Zürich, Winterthurerstraße 190, CH-8057 Zürich 


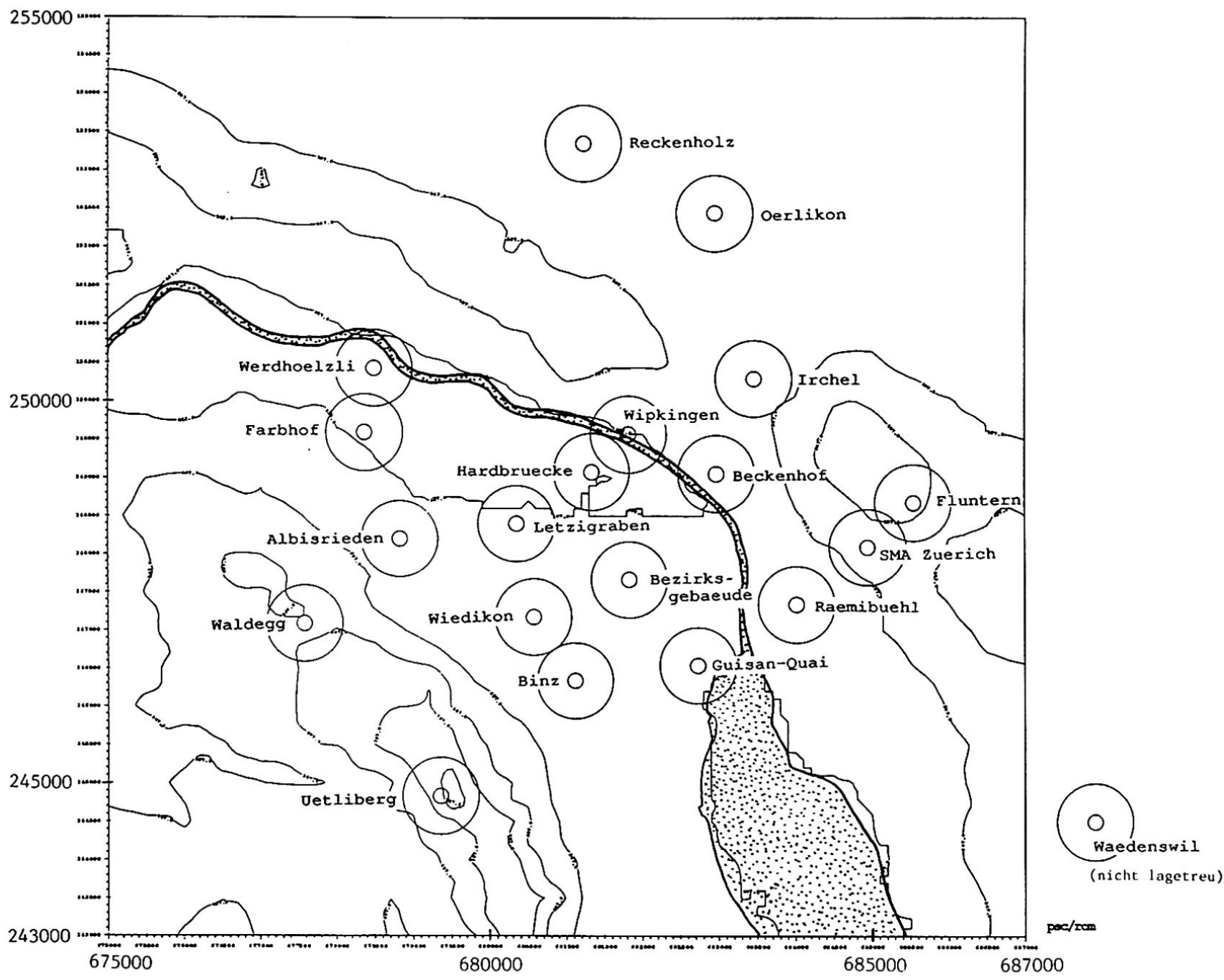

Fig.1 Meteorologisches Meßnetz im Projekt STADTKLIMA Zürich.

Meßnetz wurden auch Messungen im Frühsommer, von April bis Juni 1987, durchgeführt.

Das Meßnetz war somit auf einen mesoskaligen Maßstab ausgelegt, der das Stadtgebiet als Ganzes umfaßte und nicht einzelne Straßenzüge. Von diesen untergeordneten Strukturen interessierten lediglich diejenigen, die möglicherweise eine gesamtstädtische Bedeutung aufwiesen. Aus dem Blickwinkel der Stadtentwicklung kam dabei vor allem die Schneise in Frage, welche durch die SBBGeleise vor dem Hauptbahnhof gebildet wird.

Während einer kurzen Zeit wurden zusätzliche Meßgeräte direkt im Schienenraum eingesetzt, und zwar wiederum nicht am Boden, sondern über Dachniveau. Die Standorte befanden sich auf der Hardbrücke, auf einer Signalbrücke unmittelbar vor den Perronhallen des Hauptbahnhofes sowie auf einem Gebäude bei der Bahnhofbrücke, in der Verlängerung der beiden anderen Standorte.
Von diesen kurzzeitigen Messungen im Schienenraum erwartete man Hinweise zu folgender Frage: Gibt es ein Signal, wonach sich die Windverhältnisse im Schienenraum mindestens zeitweise deutlich vom übrigen Windverhalten unterscheiden?

\section{Untersuchungsmittel zur Windfeldanalyse}

\subsection{Einheit}

Die Untersuchungseinheit, auf deren Basis die Windfeldmessungen analysiert wurden, war die Wetterepisode. Eine Episode wurde definiert als Zeitspanne, während deren gleiche synoptische Verhältnisse herrschen. Zur Identifikation wurde die klimatologische Einteilung der Witterungslagen nach Schüepp der Alpenwetterstatistik verwendet (SMA, 1985). 


\subsection{Graphische Hilfsmittel}

Die Meßergebnisse der einzelnen Episoden wurden auf zwei Arten graphisch analysiert: Die Windrose gibt einen Überblick über die registrierten Einzelereignisse. Die Richtungen der Sektoren zeigen die Herkunft des Windes an, die Länge der Sektoren gibt die Häufigkeit an, mit der die entsprechende Windrichtung aufgetreten ist. Die Windrose sagt nichts darüber aus, wann innerhalb der Episode die Registrierung erfolgte.

Zur Analyse der zeitlichen Variabilität wurde deshalb zusätzlich der mittlere Tagesgang der Windvektoren berechnet. Die Vektoren zeigen in Strömungsrichtung und repräsentieren den mittleren Massenfluß. Zugleich erhält man eine Filterung gegenüber den registrierten Einzelwerten.

\subsection{Diagnostisches Windfeldmodell}

Die flächendeckenden Windfelder werden numerisch mit einem diagnostischen Modell (DIAMO) berechnet, das den Einfluß der Topographie berücksichtigt. Eingangsgrößen in das Modell sind die Vektoren des mittleren Massenflusses zu einer bestimmten Uhrzeit. Das Ergebnis ist daher primär von den gemessenen Eingabedaten abhängig.

Der mathematische Hintergrund des Modells basiert auf der Publikation von SHERMAN (1978) und führt auf eine Poisson-Gleichung. DIAMO berücksichtigt die Topographie durch die Verwendung von geländefolgenden Koordinaten, die in der Vertikalen eine logarithmische Einteilung aufweisen. Die mathematischen und numerischen Aspekte sowie Testergebnisse von DIAMO sind in SCHUHMACHER (1991) beschrieben.

\subsection{Numerisches Strömungsmodell}

In einem Forschungsprojekt interessieren immer auch die theroretischen Hintergründe, die zu einem bestimmten Phänomen führen. Das «abgeschlossene Labor» des Klimatologen, in dem einzelne Prozesse identifiziert werden können, ist das numerische Modell. Im vorliegenden Projekt wurde ein Computermodell entwickelt und eingesetzt, das aufgrund der theoretischen Gleichungen die dreidimensionale Strömung über Topographie berechnet. Der Programmcode MONARC (Model for Non-hydrostatic Atmospheric Research Computations) ist als nichthydrostatisches Modell konzipiert. Die Topographie wird wie bei DIAMO mit geländefolgenden Koordinaten berücksichtigt. Die Gleichungen werden an Gitterpunkten gelöst, wobei die Druck- und Geschwindigkeitsknoten aus Gründen der numerischen Stabilität gegeneinander verschoben sind (staggered grid). Die zeitliche Integration erfolgt mit einem impliziten Verfahren, das die dreidimensionalen Gleichungen in drei eindimensionale Räume aufteilt (ADI = alternativ directing implicit-Methode). Die mathematischen und numerischen Aspekte von MONARC sind in SCHUHMACHER (1991) beschrieben.

\section{Vergleich zwischen Beobachtung und Berechnung mit dem numerischen Modell anhand einer Ostlage}

Die Ostlage vom 23. bis 25.2.1987 ist gekennzeichnet mit einer deutlichen Temperaturinversion. Die Untergrenze liegt um $1700 \mathrm{~m}$ ü. M., die Inversionsstärke beträgt etwa 8 K. Unterhalb der Inversion erfolgt eine Windanströmung aus $60^{\circ}$ bis $30^{\circ}$, mit einer Geschwindigkeit von 6 $\mathrm{m} / \mathrm{s}$. Mit dem numerischen Modell MONARC wird untersucht, inwieweit allein die Topographie ohne städtische Einflüsse das Bodenwindfeld der Region Zürich modifiziert. Die synoptische Lage dieser Episode wird als Anfangszustand vorgegeben. Der untere Modellrand wird durch die digitalisierte Topographie gebildet, die mit einer Maschenweite von $500 \times 500$ m keine Stadtstrukturen aufweist. Die Bodentemperatur wird im ganzen Modellgebiet einheitlich mit 271,5 K vorgegeben und ist um $0,5 \mathrm{~K}$ kühler als die Luftemperatur, die bis $1700 \mathrm{~m}$ ü. M. neutral geschichtet ist.

Bei der zeitlichen Integration der Modellgleichungen werden die Randbedingungen nicht verändert. Nach einer Integrationszeit von $5 \mathrm{~h}$ ergibt sich ein stationäres Windfeld, das nicht mehr von den Anfangsbedingungen abhängig ist. Das Bodenwindfeld auf etwa 35 m über Grund ist in Figur 2 dargestellt.

Die stabile Strömung fließt von NE her ins Stadtgebiet ein. Sie erhält eine starke Drehung bereits über dem Stadtgebiet, da die Luftmassen die Flanken des Üetliberges nicht überströmen können. Die Strömung verläßt daher das Stadtgebiet in SSE-Richtung.

Die Messungen dieser Episode sind als Windrosenkarte in Figur 3 dargestellt. Dabei sind nicht alle Stationen eingetragen. Weitere, die ebenfalls in Betrieb waren, gehören zum «Experiment Schienenraum», worauf später einge-

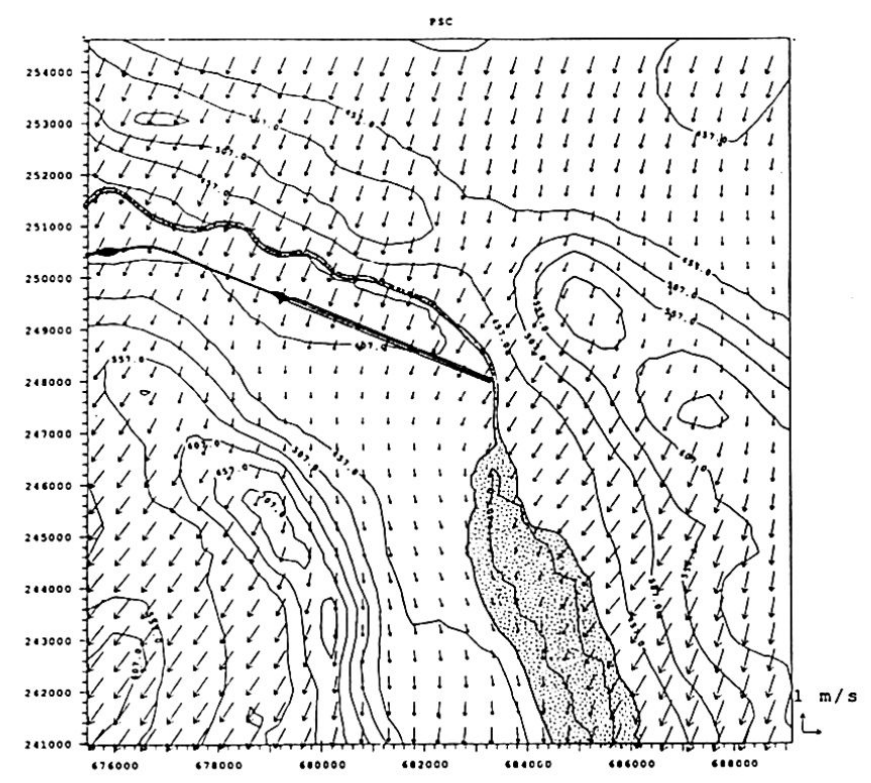

Fig. 2 Ostlage: Mit MONARC modelliertes Bodenwindfeld. 


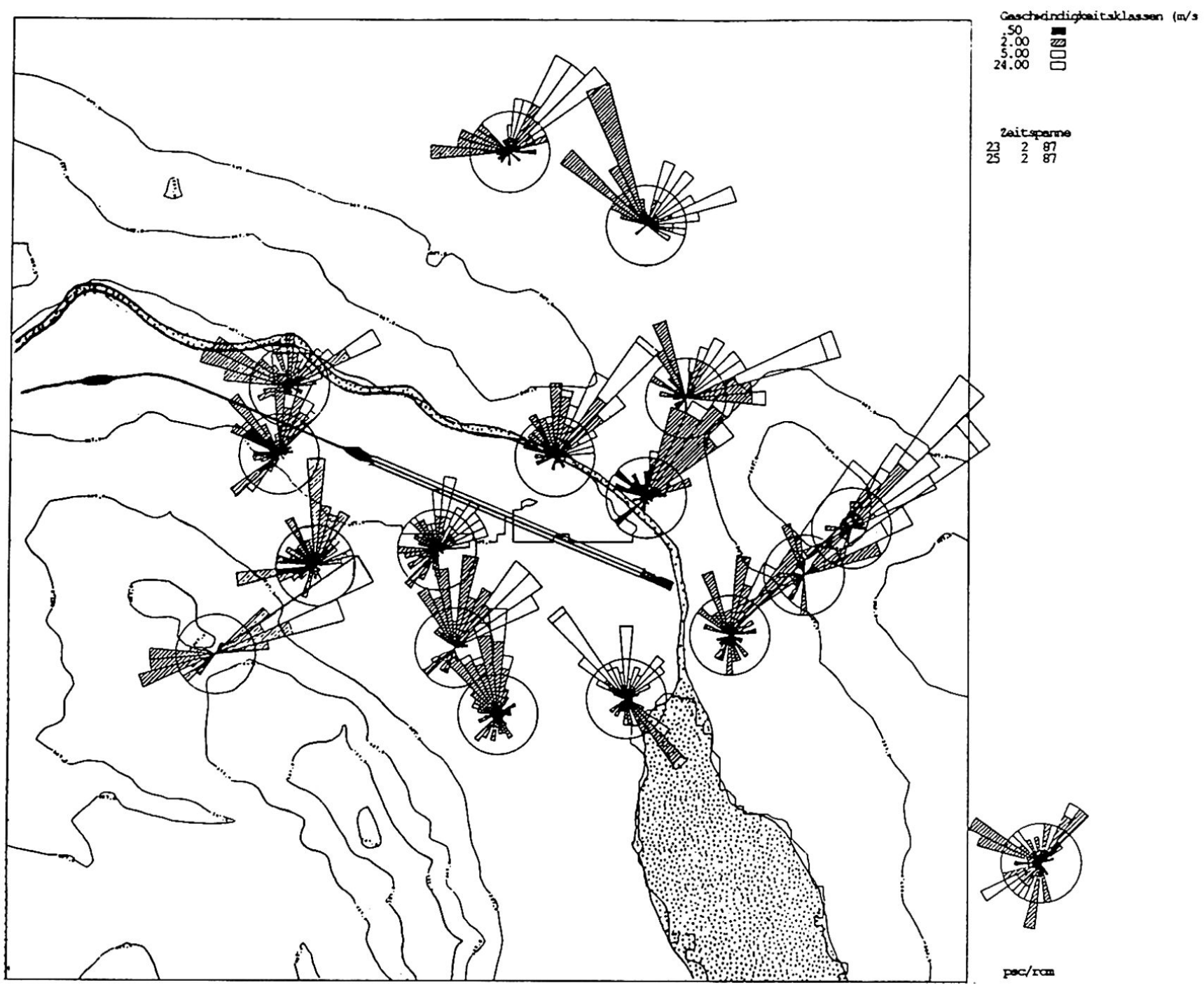

Fig. 3 Ostlage: Windrosenkarte der Episode vom 23. bis 25.2.1987.

gangen wird. Die Analyse des zeitlichen Ablaufs ergibt, daß bei den Stationen im Norden und Westen die nordwestlichen Richtungssektoren den Nacht- und Vormittagsstunden zuzuordnen sind, die nordöstlichen den Nachmittagsstunden.

Um einen Vergleich mit den Modellergebnissen von MONARC ziehen zu können, wird aus den Meßergebnissen mit dem Modell DIAMO ein diagnostisches Windfeld berechnet. Als Eingangsgrößen dienen von jeder Meßstation der mittlere Massenflußvektor um 11 Uhr. Das entsprechende Bodenwindfeld ist in Figur 4 dargestellt und repräsentiert die Verhältnisse zwischen 5 und 11 Uhr morgens. Gut erkennbar ist auch hier das Einströmen aus NE, die Drehung über der Stadt und das Ausströmen in südlicher Richtung.

Zwischen dem beobachteten und dem numerisch berechneten Windfeld besteht bereits eine recht gute Übereinstimmung, ohne daß in MONARC die Stadt speziell berücksichtigt worden war. Ähnliches konnte auch bei anderen synoptischen Anströmungen gefunden werden.
Die Folgerung aus dem numerischen Ergebnis ist, daß die Form der Topographie zusammen mit der atmosphärischen Stabilität primäre Einflußfaktoren für das Bodenwindfeld in Zürich sind.

\section{Mesoskalige und mikroskalige Windstrukturen am Beispiel des Schienenraumes}

Während der gleichen Ostlage vom 23. bis 25.2 .1987 befanden sich weitere Stationen in Betrieb. Wie die übrigen Stationen befanden sie sich nicht auf Bodenniveau, sondern ebenfalls über Dachniveau. Diese drei Stationen lagen jedoch alle auf einer Gerade über dem Schienenraum, der vom Limmattal in den SBB-Hauptbahnhof führt. Die Geleiseanlagen weisen dort eine Breite von über $250 \mathrm{~m}$ auf und sind seitlich begrenzt mit Häusern, deren Höhen zwischen $20 \mathrm{~m}$ und $30 \mathrm{~m}$ liegen. Es handelt sich also nicht um eine enge "Geleiseschlucht», sondern eher um eine breite Senke. Ob hier eine mikroskalige 


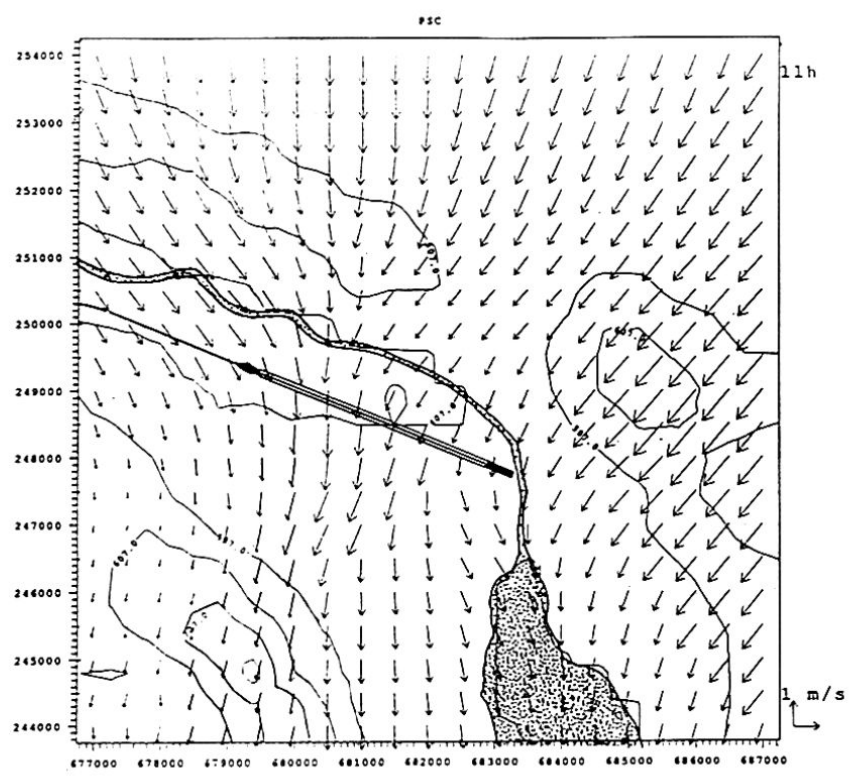

Fig. 4 Ostlage: Diagnostisches Windfeld, berechnet mit DIAMO aufgrund von gemessenen Winddaten.
Windfeldänderung auftritt, die auch in der Mitte des Geleiseraumes auf Höhe des seitlichen Dachniveaus registriert werden kann, ist somit nicht zum vornherein klar. Die entsprechende Windrosenkarte, wo auch die Stationen im Bereich des Schienenraumes eingetragen sind, ist in Figur 5 dargestellt. Diese Stationen weisen Windrichtungen auf, die parallel zum Geleiseareal verlaufen. Vergleicht man das neu berechnete diagnostische Windfeld in Figur 6 mit der Situation, wo die Stationen im Schienenraum nicht berücksichtigt wurden (Fig. 4), so stellt man deutlich eine "Störung» fest, die den Schienenraum andeutet. Das mikroskalige Strömungsverhalten im Geleiseareal kann in diesem Maßstab auch diagnostisch nicht korrekt berechnet werden, da mit einer Maschenweite von $500 \mathrm{~m}$ der Schienenraum nicht erfaßt werden kann.

Aus diesem Experiment «Schienenraum» können fünf Folgerungen abgeleitet werden:

1. Das kurzzeitige Experiment gab einen klaren Hinweis, daß im Schienenraum andere Windstrukturen herrschen können als im übrigen mesoskaligen

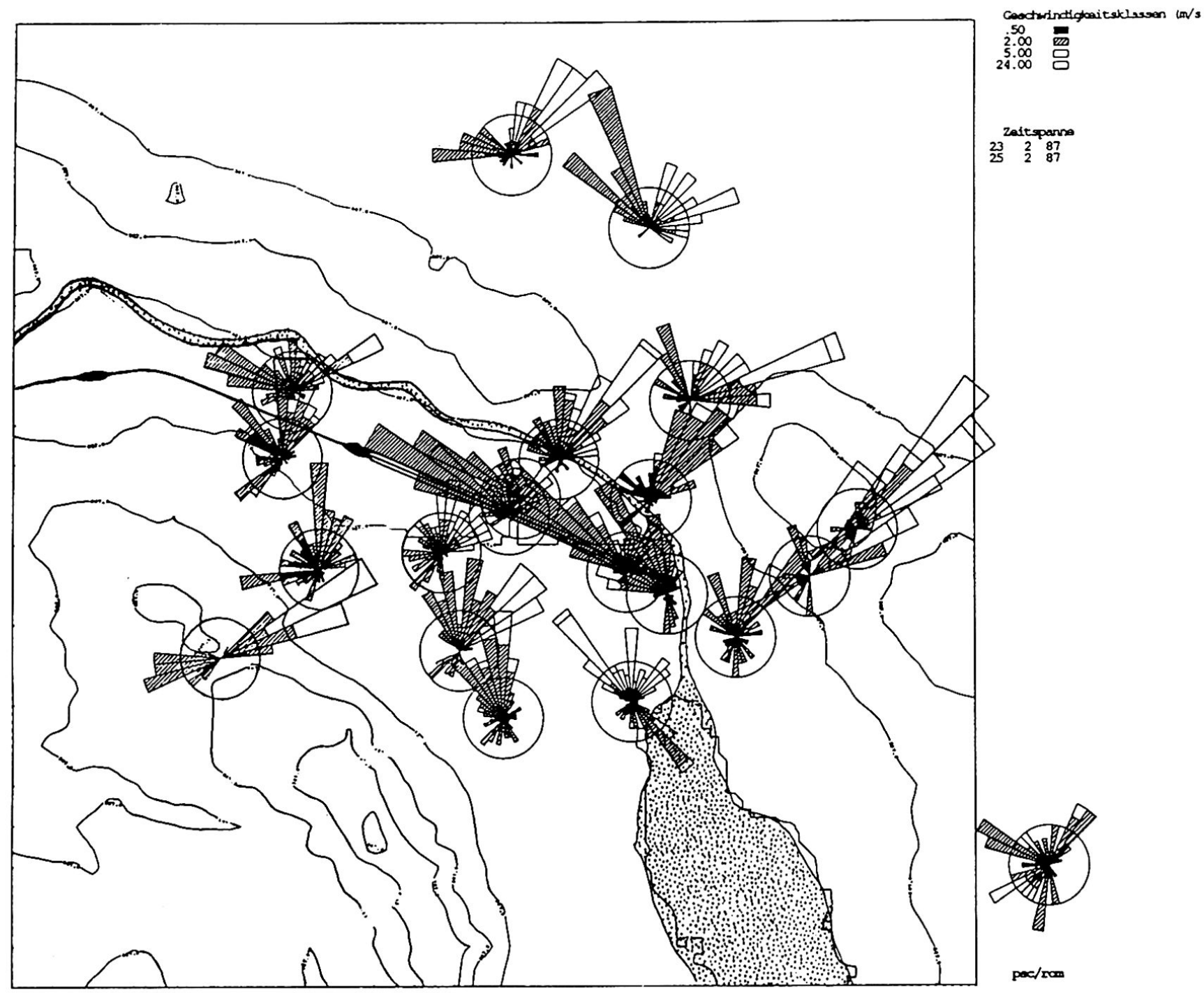

Fig. 5 Wie Abbildung 3, jedoch mit Berücksichtigung der Stationen im Bereich des Schienenraumes. 


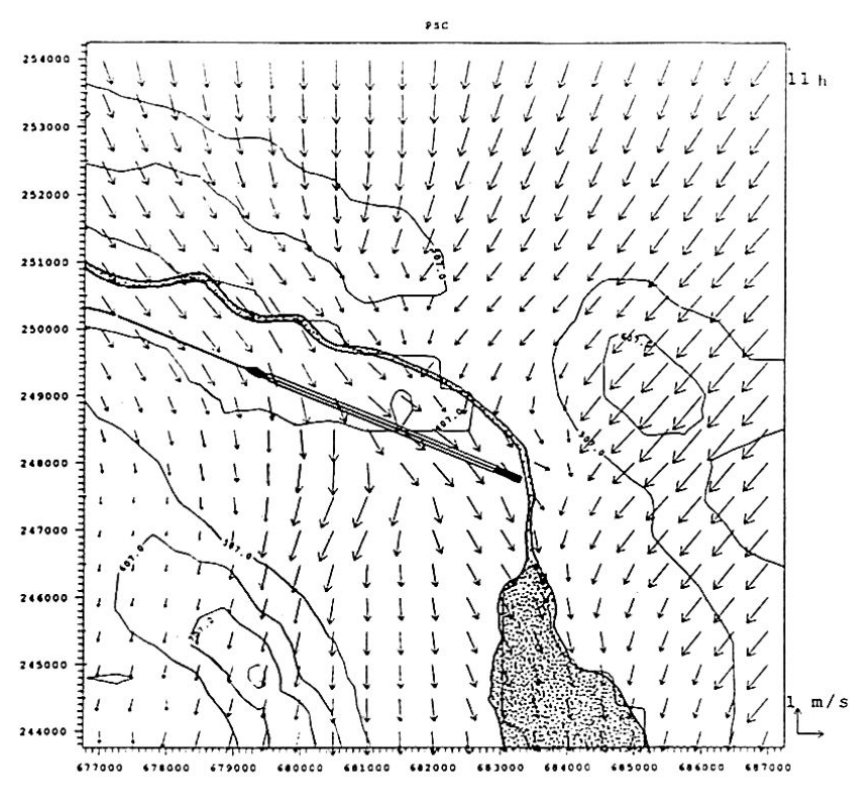

Fig. 6 Wie Abbildung 4, jedoch mit Berücksichtigung der Stationen im Bereich des Schienenraumes.

Windfeld der Region Zürich. Dieses Signal war in einer Situation zu finden, wo die mesoskalige Strömung ungefähr senkrecht zur Schienenrichtung verlief. Die Messungen im Schienenraum selber zeigten eine fast rechtwinklige, mikroskalige Abweichung dazu.

2. Die Relevanz für dieses Phänomen müßte mit einer längeren Meßreihe überprüft werden.

3. Ohne die entsprechenden Messungen sind Aussagen zum mikroskaligen Windfeldverhalten im Schienenraum nicht möglich.

4. Die Ergebnisse zeigen, daß für eine derartige Messung sowohl Mikroskala (Schienenraum) wie Mesoskala (Region Zürich) gleichzeitig adäquat erfaßt werden müssen, da sonst keine Interpretation möglich ist.

5. Die numerische Modellierung ist ein wichtiges Hilfsmittel zur Interpretation. Aber auch hier müssen die verschiedenen Skalen sowohl bezüglich der räumlichen Auflösung als auch bezüglich der physikalischen Formulierung berücksichtigt werden.

\section{Konvergentes Windfeld und Schadstofftransport gezeigt anhand einer Westlage}

Synoptische Wetterlagen mit Strömungen aus West und Südwest treten sehr häufig auf. Aufgrund der meist gröBeren Windgeschwindigkeiten werden dann Lokalzirkulationen weniger erwartet. Aus der Analyse der Meßergebnisse zeigte sich aber, daß bei verschiedensten Stabilitäts- und Windgeschwindigkeitsverhältnissen in Zürich dann deutlich konvergente Windmuster auftreten, indem der Wind gleichzeitig sowohl vom See als auch vom Limmattal her gegen das Stadtzentrum gerichtet ist. Systematische Untersuchungen mit MONARC ergaben, daß dazu verschiedene Gründe verantwortlich sind. Bei synoptischen Winden aus West und Südwest steht der Üetliberg quer als Riegel in der Strömung und schirmt die Stadt Zürich ab. Ein erster wichtiger Faktor ist nun die atmosphärische Stabilität und die genaue Anströmungsrichtung, die entscheiden, ob sich in Zürich am Boden eine generelle SE- oder NW-Strömung einstellt, oder - durch die beidseitige Umströmung des Üetliberges - beides gleichzeitig. Ein zweiter Faktor ist die städtische Wärmeinsel, die die Winde in Richtung Stadtzentrum ablenkt. Mit zunehmender Windgeschwindigkeit tritt ein dritter Faktor auf, indem die Strömungsdynamik im Lee des Üetliberges zu einem leichten Unterdruck führt.

Alle drei Faktoren haben die Tendenz, in Zürich konvergente Strömungen hervorzurufen, was Konsequenzen für die Schadstoffverteilung hat. Ein typisches konvergentes Bild zeigt die Westwindepisode vom 21.6.1987 bis 26.6.1987, von der in Figur 7 ein diagnostisches Windfeld gezeigt ist. Diese Grundstruktur bleibt den ganzen Tag über erhalten. Dieses Windfeld wurde als Eingangsgröße für das Lagrangesche Diffusions- und Trajektorienmodell LADI-TRAMO verwendet. Zur Illustration werden an zwei fiktiven Quellen Schadstoffemissionen abgegeben und durch die turbulente Modellatmosphäre transportiert. Die Schadstoffpartikeln werden durch das konvergente Strömungsmuster zuerst ins innere Stadtgebiet hinein getragen, bevor sie gemeinsam durch die Paßlage am Milchbuck in die Außenquartiere gelangen.

Folgende Schlüsse sind zu ziehen: Aus verschiedensten Prozessen treten im Stadtgebiet von Zürich bei Westund Südwestströmungen häufig konvergente Windfelder auf. Dadurch wird die lufthygienische Situation ver-

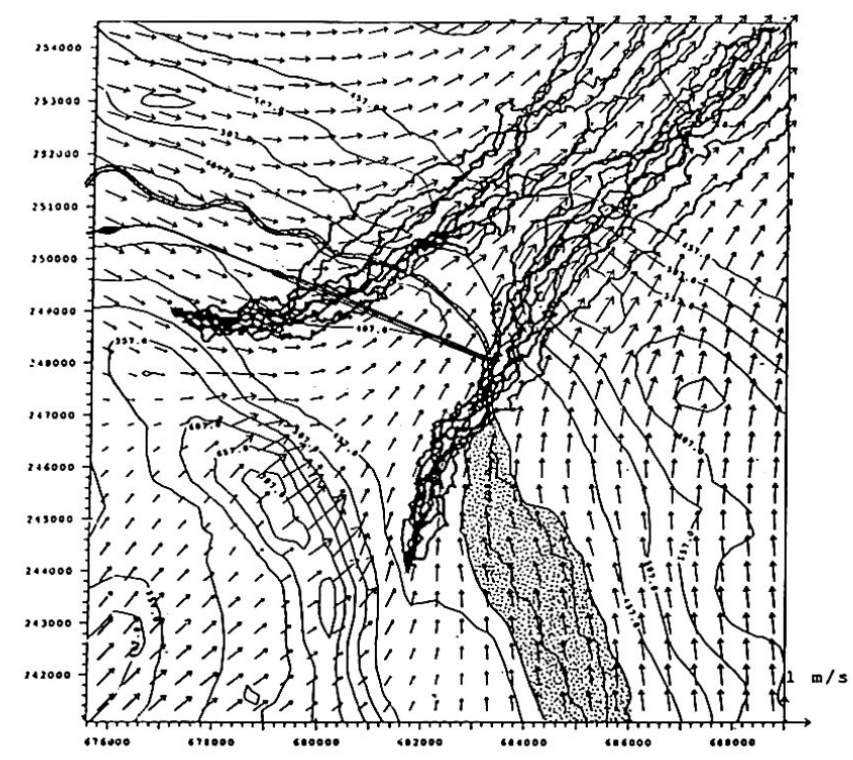

Fig. 7 Westlage: Diagnostisches Windfeld, berechnet aufgrund der Messungen vom 21. bis 26.6.1987. Trajektorien von zwei fiktiven Schadstoffquellen, berechnet mit einem Partikeltrajektorienmodell (LADI-TRAMO). 
schärft. Vom modelltechnischen Standpunkt aus bedeutet dies, daß Immissionsmodelle eingesetzt werden müssen, welche diese Situationen auch abbilden können.

\section{Verdankung}

Das Projekt wurde durch den Schweizerischen Nationalfonds zur Förderung der wissenschaftlichen Forschung finanziert (Nr. 2.580-0.84 und 2.220-086). Die Schweizerische Meteorologische Anstalt Payerne (Sektion Umweltmeteorologie) unterstützte das Projekt in Form von technischer Zusammenarbeit ebenfalls wesentlich.

Prof. Dr. A. Ohmura, ETH Zürich, danke ich für die Durchsicht dieses Artikels, Frau P. Schibli, Architektin, Wettingen, für die Erstellung der Grundlagenkarten und Frau M. Werfeli, ETH Zürich, für die sorgfältige Reinschrift.

\section{Literatur}

BENZ, U. (1988): Parametrisierung der planetaren Grenzschichthöhe über der Stadt Zürich. Diplomarbeit Geographisches Institut der ETH Zürich, 130 p.
GYSEL, P. (1984): Vergleich der 4 Komponenten der Strahlungsbilanz am Boden zwischen Albiskette und Stadtzentrum - Rückschlüsse auf den Aerosoleinfluß. Diplomarbeit Labor für Atmosphärenphysik der ETH Zürich, 77 p.

HOFER, B. (1985): UV-Strahlung im Stadtzentrum von Zürich. Diplomarbeit Geographisches Institut der ETH Zürich, $121 \mathrm{p}$.

LEUTENEGGER, T. (1988): Modellierung der kurzwelligen Strahlungsbilanz in einem Straßencanyon. Diplomarbeit Geographisches Institut der ETH Zürich, 46 p.

ROTH, M. (1985): Albedomessung über dem Schweizerischen Mittelland. Diplomarbeit Geographisches Institut der ETH Zürich, $130 \mathrm{p}$

ROTACH, M. (1990): Turbulence within and above an urban canopy. Dissertation in Vorbereitung am Geographischen Institut der ETH Zürich.

SCHÄDLER, G. (1988): Windprofile über Zürich: Drei Orte im Vergleich. Diplomarbeit Geographisches Institut der Universität und der ETH Zürich, $100 \mathrm{p}$.

SCHUHMACHER, P. (1991): Messungen und numerische Modellierungen zum Windfeld über einer Stadt in komplexer Topographie. Dissertation ETH Zürich Nr. 9390.

SHERMAN, C.A. (1978): A mass-consistent model for windfields over complex terrain, J. Appl. Meteor., 17, 312-319.

SMA (1985): Alpenwetterstatistik, Witterungskalender, Schweizerische Meteorologische Anstalt, Zürich. 